\title{
REPERCUSSÕES CLÍNICAS ASSOCIADAS À ESQUIZOFRENIA INFANTIL: RELATO DE CASO
}

\author{
CLINICAL REPERCUSSIONS ASSOCIATED WITH CHILDHOOD \\ SCHIZOPHRENIA: CASE REPORT
}

\author{
Carolina Campos Ribeiro ${ }^{1 *}$, Érica Zerbone Santanna ${ }^{1}$, Esther de Souza Beiral ${ }^{1}$, Beatriz Rangel Rufino ${ }^{1}$, Camila Gomes \\ Marques $^{2}$, Shaytner Campos Duarte ${ }^{3}$, Leonardo Muniz Soares Dias Duarte ${ }^{4}$ \\ *1. Graduanda em Medicina pela Faculdade de Medicina de Campos, 10 período, (carol.camposribeiro@gmail.com); \\ 2. Graduada em Psicologia pela Universidade Federal Fluminense. Atuante em Psicologia Clínica; \\ 3. Professor Doutor da Faculdade de Medicina de Campos; \\ 4. Psiquiatra Infantil e Professor da Faculdade de Medicina de Campos.
}

\section{RESUMO}

Introdução: A esquizofrenia infantil é um fenômeno raro e grave, tendo como principal característica sintomas psicóticos pré-puberais ${ }^{1-3}$. Manifesta-se em menos de $1 / 40.000$ crianças $^{1-2}$ de forma insidiosa e progressiva. Entretanto, cenários pré-mórbidos são observados, como: isolamento social; atraso motor e verbal; déficit de atenção, agressividade e recusa alimentar ${ }^{1-4}$. Considerando as alterações clínicas que podem associarse a psicopatologias e/ou ao uso de psicotrópicos, em alguns casos, faz-se necessária a internação hospitalar com o objetivo de tratar tais sintomas. Os leitos psiquiátricos propostos para os Hospitais Gerais tendem a romper com o manicômio, diminuindo o estigma da doença mental; cessar a dicotomia mente/corpo proposta pelo modelo cartesiano e efetivar a Política Pública de Saúde Mental no Brasil. Objetivos: Relatar um caso de esquizofrenia infantil associado às repercussões clínicas desencadeadas pela psicopatologia de base. Expor a importância do processo de trabalho em rede e a atenção à saúde de forma integral dentro do Hospital Geral, tendo como base a Política Nacional de Saúde Mental. Descrição: Adolescente, 17 anos, pré-diagnosticada com esquizofrenia, em tratamento irregular. Apresentou há 10 dias: recusa alimentar e hídrica, isolamento social, agressividade e alterações no sono. Deu entrada no CAPSI onde foi administrado Haldol Decanoato e encaminhada para internação hospitalar, objetivando correção de distúrbios hidroeletrolíticos. Conclusão: Espera-se determinar uma variável que compreenda a condição de base de pacientes psiquiátricos e, elucide a coexistência de processos fisiopatológicos orgânicos. Desse modo, ressaltar a importância de integrar os objetivos da Reforma Psiquiátrica no que tange o acompanhamento terapêutico de pacientes implicados nesse contexto evolutivo.

Palavras-Chave: esquizofrenia infantil; psiquiatria infantil; reforma psiquiátrica; desinstitucionalização.

\section{ABSTRACT}

Introduction: The childhood schizophrenia is a rare and serious phenomenon, having as main characteristic, pre-pubertal psychotic symptoms ${ }^{1-3}$. It occurs in less than $1 / 40,000$ children $^{1-2}$ in an insidious and progressive way. However, pre-morbid scenarios are observed, such as: social isolation; motor and verbal delay; attention deficit, aggression and food refusal ${ }^{1-4}$. Considering the clinical alterations that may be associated with psychopathology and/or the use of psychotropic drugs, in some cases hospitalization is necessary to treat such symptoms. The psychiatric beds proposed for General Hospitals tend to break with the asylum, reduce the stigma of mental illness, cease the mind / body dichotomy proposed by the Cartesian model, and effect the Public Policy on Mental Health in Brazil. Objectives: To report a case of childhood schizophrenia associated with the clinical repercussions triggered by basic psychopathology. To expose the importance of the process of networking and health care in a comprehensive way within the General Hospital, based on the National Mental Health Policy. Description: Adolescent, 17 years old, pre-diagnosed with schizophrenia, in irregular treatment. Presented 10 days ago: food and water refusal, social isolation, aggression and insomnia. She was admitted to the CAPSI where Haldol Decanoato was administered and referred to the hospital to correct hydroelectrolytic disorders. Conclusion: It is expected to determine a variable that understands the basic condition of psychiatric patients, and elucidates the coexistence of organic pathophysiological processes. to emphasize the importance of integrating the objectives of the Psychiatric Reform in the therapeutic follow-up of patients in this evolutionary context.

Keywords: childhood schizophrenia; child psychiatry; psychiatric reform; deinstitutionalization. 


\section{INTRODUÇÃO}

O surgimento da esquizofrenia na infância é um fenômeno raro e grave, tendo como principal característica o surgimento de sintomas psicóticos prépuberais ${ }^{1-3}$. A esquizofrenia se manifesta em cerca de $1 \%$ da população geral, porém, na infância o cenário se modifica, sendo menos de um caso a cada $40 \mathrm{mil}$ crianças ${ }^{1-2,4}$. A esquizofrenia de início precoce ocorre de forma insidiosa e progressiva. Desse modo, a criança pode demorar meses ou anos para preencher todos os critérios diagnósticos apresentados nos manuais. Alguns sinais podem ser perceptíveis anteriormente ao quadro mórbido, como: isolamento social; habilidades sociais restritas; atraso motor e verbal; comprometimento cognitivo refletido no desempenho escolar prejudicado; déficit de atenção, aprendizado e abstração; crises de agressividade e recusa alimentar. Apesar de essas manifestações prodrômicas estarem presentes, o diagnóstico normalmente é feito após a primeira crise ${ }^{1-4}$. Considerando as alterações clínicas que podem associar-se a psicopatologias e/ou ao uso de psicotrópicos, em alguns casos, faz-se necessária a internação em hospital geral com o objetivo de tratar os sintomas correlacionados. A proposta dos leitos psiquiátricos em Hospitais Gerais tende a efetivar a Política Pública de Saúde Mental no Brasil ${ }^{5-7}$ e a romper com o manicômio, diminuindo o estigma que cerca a doença mental e cessando a ideia da dicotomia mente/ corpo estabelecida pelo modelo cartesiano.

\section{OBJETIVOS}

Relatar um caso de esquizofrenia infantil associado a repercussões clínicas desencadeadas pela psicopatologia de base. Expor a importância do processo de trabalho em rede e a atenção à saúde de forma integral dentro do Hospital Geral, tendo como base a Política Nacional de Saúde Mental.

\section{RELATO DE CASO}

Adolescente, 17 anos, sexo feminino, cor parda, natural de Campos dos Goytacazes-RJ, diagnosticada previamente com esquizofrenia. Há 10 dias, iniciou quadro de recusa alimentar e hídrica, isolamento social pronunciado, episódios de agressividade e alterações no sono. Após 4 dias, a paciente se encaminhou ao Pronto Atendimento Psiquiátrico de sua cidade e posteriormente a um Hospital Geral por duas vezes. Realizou exames laboratoriais que demonstraram hemoconcentração e uma tomografia computadorizada de crânio, que não apresentou alterações. Recebeu encaminhamento para o Centro de Atenção Psicossocial Infanto - juvenil (CAPSI), onde foi atendida por um psiquiatra infantil e uma psicóloga. Neste contexto, a mãe relatou que há 2 anos a filha apresentou um quadro semelhante ao atual e desde então, vem evoluindo com afastamento escolar e no ambiente familiar, diminuindo também condições de higiene. Nesse primeiro episódio, foi feito o diagnóstico de esquizofrenia infantil, sendo tratada com Haldol, Fenergan e Diazepam (SIC), mas no momento de atendimento no dispositivo, o uso das medicações havia sido interrompido. Ao exame físico, a paciente encontrava-se entristecida, com vestes mal compostas, indiferente, não cooperativa, anedônica, com mutismo, hipocorada (+/4+), desidratada, e com PA: 90 × 60 mmHg. O psiquiatra infantil optou por fazer Haldol Decanoato $50 \mathrm{mg} / \mathrm{ml}$ via intramuscular. Quando foi admitida no Hospital Plantadores de Cana, profissionais do CAPSI que acompanhavam o caso foram visitá-la, tendo como conduta inicial ouvir as demandas da paciente, acolher os familiares e conversar com a equipe hospitalar responsável no dia. A paciente estava acompanhada pelo pai, falou pouco, mas manifestou desejo em ir ao banheiro e tomar banho, além de enfatizar que não se sentiu bem com a forma com que foi tratada em outra instituição hospitalar - contenção em maca -, temendo retorno a mesma. Neste caso, foi explicado que a mudança de hospital tinha como principal objetivo que a equipe envolvida no caso conseguisse dar suporte necessário e acompanhar de perto todas as condutas tomadas. Ao exame clínico, em dia posterior, referia tosse produtiva com saída de secreção esverdeada e um episódio febril no dia anterior (Temperatura axilar: $37,6^{\circ} \mathrm{C}$ ). Ao exame físico, apresentava bom estado geral, desidratada, hipocorada $(+/ 4+)$, alimentando-se com maior facilidade com preferência por alimentos líquidos e pastosos e na ausculta pulmonar, identificados estertores crepitantes em base de hemitórax direito. Foram solicitados exames laboratoriais na admissão: leucograma (16.370); Hemoglobina (13,2); Hematócrito $(36,4 \%)$; Plaquetas (262.000); Ureia (17); Creatinina (0.6); $\mathrm{Na}^{+}$ (128); $\mathrm{K}^{+}(2,7)$; Glicose (157); $\mathrm{Ca}^{2+}(9,1) ; \mathrm{Mg}^{2+}(1,8)$; $\mathrm{P}^{-}(2,0)$. Raio-X de tórax evidenciando infiltrados intersticiais em base pulmonar direita. A conduta traçada foi: dieta hiperproteica e hipercalórica, hidratação venosa associada à reposição de potássio (2 ampolas de $\mathrm{KCl} 10 \%$ ) e complexo B (2 ampolas), sintomáticos, Haldol $5 \mathrm{mg}$ via oral, Fenergan $25 \mathrm{mg}$ via oral - devido a episódio de rigidez muscular identificada durante a internação hospitalar, 
provavelmente pelo uso do Haldol - e Ampicilina 1g via venosa devido ao quadro de pneumonia atípica apresentada pela paciente. $\mathrm{O}$ atendimento realizado, bem como a conduta expressa, foram informados a todos que estavam acompanhando o caso, facilitando intervenções seguintes. No atendimento realizado pela profissional de Psicologia, a paciente encontrava-se muito sonolenta e com dificuldades para conversar, o acolhimento foi então realizado com a mãe, possibilitando o relato de que algumas características da paciente podem ser originárias da relação familiar, atribuindo alguns fatores à manifestação do quadro apresentado. A mãe informou que a filha sempre foi retraída e evitava conversar com muitas pessoas, inclusive familiares. Em alguns momentos, escrevia o que desejava expressar, semelhante ao que fez no início desta crise quando foi interrompendo a comunicação verbal. A paciente sempre criticava algumas condutas do pai e por este motivo, a relação entre eles não poderia ser considerada saudável, sendo marcada por discussões constantes e afastamento (SIC). Na primeira manifestação do quadro de esquizofrenia, o pai percebeu que sua conduta poderia ser um fator complicador para a saúde mental da filha e se propôs a modificar. Segundo a mãe, conseguiu agir diferente durante um tempo, mas atualmente estava muito nervoso e agredia a família verbalmente com frequência significativa. Após 5 dias de internação hospitalar, a paciente evoluiu com melhora clínica, aceitando bem a dieta e com permanência da tosse, porém, em menor frequência e com saída de secreção clara. Realizou novos exames laboratoriais para controle da correção dos distúrbios hidroeletrolíticos: Leucograma (9.030); Hemoglobina $(12,8)$; Hematócrito (36,4\%); Plaquetas (347.000); $\mathrm{Na}^{+}(138)$; $\mathrm{K}^{+}(3,6)$. Foi dada alta hospitalar com prescrição de Haldol $5 \mathrm{mg}$, Fenergan $25 \mathrm{mg}$ e Azitromicina $500 \mathrm{mg}$ por 5 dias, bem como o encaminhamento para o CAPSI, onde foi dada continuidade ao atendimento da paciente de forma psicossocial. No dia da alta, a equipe interdisciplinar que atuou no HPC e no CAPSI estava presente, sendo possível passar as informações necessárias à paciente e à sua família, enfatizando a necessidade de dar continuidade ao acompanhamento da mesma.

\section{DISCUSSÃO}

Ao avaliar a adolescente em um primeiro atendimento, a mesma encontrava-se com características clínicas de evolução insidiosa de distanciamento social, deterioração do desempenho escolar e das atividades cotidianas há 3 meses. No último mês, houve um agravamento desses sintomas associados a franco prejuízo emocional e clínico, devido à importante recusa alimentar, tanto de alimentos sólidos quanto líquidos. Há também piora de sua autonomia de cuidado pessoal com recusa a tomar banho, escovar os dentes e trocar de roupa, bem como de outras atividades de vida diária. Concluindo esse primeiro relato, a família descreve características de afeto inapropriado e delírios persecutórios. Ao questionar sobre queixas comportamentais prévias, houve um relato, pela mãe, de um contexto semelhante, há cerca de 2 anos, associado a episódios de agressividade, fuga, agitação psicomotora, além do comportamento de escrever seus desejos em papel quando tinha preferência por evitar o contato interpessoal. Neste episódio, apresentou considerável resposta clínica frente ao uso de Haldol $5 \mathrm{mg}$ via oral 12/12h. Entretanto, estava sem fazer uso de medicações durante o primeiro atendimento no CAPSI. Com a história clínica apresentada no relato acima, foi aventada a hipótese diagnóstica de esquizofrenia infantil. Diante disso, pela impossibilidade de ingestão, devido à recusa de substâncias sólidas e líquidas, a via intramuscular foi a escolhida para a administração de um antipsicótico típico, como o Haldol Decanoato $50 \mathrm{mg} / \mathrm{ml}$ e posterior encaminhamento para internação hospitalar com o objetivo de estabilizá-la clinicamente devido às repercussões orgânicas associadas ao fator causa-efeito inserido no contexto psíquico no qual a mesma se encontrava. Após 12 horas de internação hospitalar - na qual, no momento da admissão, foi administrado Haldol $5 \mathrm{mg}$ por via oral conforme a aceitação da paciente - e aproximadamente 36 horas após administração do Haldol Decanoato, foi observada uma melhora da incoerência do pensamento, que se refletiu na restauração gradual do contato social, fala, do autocuidado e da recuperação de apetite. Estes fatores já citados permitiram que a intervenção realizada pela equipe ao chegar ao hospital neste primeiro dia de internação pudesse ser efetivada, entendendo os desejos e as queixas da paciente pela via da escuta. Além da questão meramente psíquica na qual a paciente encontrava-se, as repercussões clínicas geradas por essa interrupção da ingesta hídrica e alimentar podem ser destacadas: desidratação, hipocalemia, hiponatremia, e também, um quadro infeccioso compatível com pneumonia atípica - através de sinais clínicos, laboratoriais e radiológicos -. Algumas dificuldades foram encontradas no que diz respeito ao cuidado e admissão de um paciente 
psiquiátrico no Hospital Geral, corroborando com o movimento de luta pela desinstitucionalização. A disponibilidade de profissionais que atuassem nos dois ambientes em que a paciente estava inserida e em acompanhamento efetiva o movimento de rede de atenção psicossocial, neste caso manifestado em uma situação de urgência. $\mathrm{O}$ movimento instituído permitiu um atendimento e acolhimento integral à paciente, abrangendo sua rede de apoio, além de permitir o contato com outros profissionais que atuam no Hospital Geral, facilitando trocas e exposição da proposta da Política Pública de Saúde Mental do país.

\section{CONCLUSÃO}

Espera-se determinar uma variável que compreenda não somente a condição de base de pacientes psiquiátricos, mas que também seja capaz de elucidar a possibilidade de coexistência de processos fisiopatológicos orgânicos. Desse modo, ressaltar a extrema importância de integrar os principais objetivos da Reforma Psiquiátrica no que tange o acompanhamento do manejo terapêutico - tanto clínico-médico, quanto psicológico - de pacientes implicados nesse mesmo contexto evolutivo.

\section{REFERÊNCIAS BIBLIOGRÁFICAS}

1. SADOCK BJ, SADOCKVA. Esquizofrenia de início precoce. In: . (Org). Manual conciso de Psiquiatria da Infância e Adolescência. Porto Alegre: Artmed, 2011.p. 176-180.

2. SADOCK BJ, SADOCK VA, RUIZ P. Psiquiatria Infantil. In: 2017.p. 1268-1273.

3. ASSUMPÇÃO FB, KUCZYNSKI E. Esquizofrenia Infantil. In: ROSENBERG R. Tratado de Psiquiatria da Infância e da Adolescência. $2^{\text {a }}$ ed. São Paulo: Atheneu; 2012. p. 319-327.

4. TENGAN SK, MAIA AK. Psicoses funcionais na infância e adolescência. J Pediatr (Rio J), v.80(5), 2004. 5. BOTEGA NJ, DALGALARRONDO P. Saúde mental no hospital geral: espaço para o psíquico. 2.ed. São Paulo: Hucitec, 1997;

6. DAMÁSIO A. O Erro de Descartes: Emoção, Razão e o Cérebro Humano. $2^{\text {a }}$ ed. São Paulo: Companhia das Letras; 1999; 7. MION JZ, SCHNEIDER JF. Leitos Psiquiátricos em hospital geral: visão de profissionais que atuam em hospital geral. Revista Eletrônica de Enfermagem, v.5:38-42, 2003. 